Case report

\title{
A fatal case of valproate-induced hyperammonemic encephalopathy: An update on proposed pathogenic mechanisms and treatment options
}

\author{
Yeung Gloria Wai Yue ${ }^{\mathrm{a}, *}$, Chau Kei Wai ${ }^{\mathrm{b}}$, Woo Yat Ming Peter ${ }^{\mathrm{a}}$, Wong Hoi Tunga \\ Chan Kwong Yau ${ }^{\mathrm{a}}$ \\ a Department of Neurosurgery, Kwong Wah Hospital, 25 Waterloo Road, Hong Kong Special Administrative Region \\ ${ }^{\mathrm{b}}$ Li Ka Shing Faculty of Medicine, The University of Hong Kong, Hong Kong Special Administrative Region
}

\section{A R T I C L E I N F O}

\section{Article history:}

Received 24 May 2017

Accepted 13 September 2017

Available online 14 September 2017

\section{Introduction}

Sodium valproate is widely used in a range of disorders including seizures, psychiatric conditions and chronic pain syndromes. Although it has a relatively favourable safety profile, it is associated with severe idiosyncratic adverse effects, the most notable being valproate-induced hyperammonemic encephalopathy (VHE), a rare phenomenon with fewer than 150 cases documented in the last 10 years. ${ }^{1}$ The clinical presentation of VHE may be subtle and if left untreated also fatal. ${ }^{2,3}$ This case report illustrates the importance early recognition and management of this rare and lethal adverse effect.

\section{Case report}

A 71-year-old female, with known hypertension and ChildPugh A Hepatitis B viral chronic liver disease was admitted for sudden expressive dysphasia. Upon admission the patient's Glasgow Coma Scale (GCS) score was 11. Initial blood results including liver function were within normal limits. A computed tomography brain scan (CTB) revealed a left frontal lobar intracerebral hematoma measuring $2.3 \mathrm{~cm} \times 1.5 \mathrm{~cm} \times 2.5 \mathrm{~cm}$ (volume: $4.5 \mathrm{ml}$ ) and Sylvian fissure subarachnoid hemorrhage (SAH).

Soon after admission, the patient developed status epilepticus. She was mechanically ventilated and resuscitated. In addition to the administration of intravenous midazolam, sodium valproate ( $400 \mathrm{mg}$, eight-hourly) was administered with successful cessation of seizures. In view of a suspected ruptured intracranial aneurysm and the risk of delayed ischemic neurological deficit, early systemic nimodipine was started according to our institution's protocol.

\footnotetext{
* Corresponding author.

E-mail address: gloria.yeung@outlook.com (G.W.Y. Yeung).
}

Valproate was selected as it is the only widely available broad spectrum antiepileptic proven to be similar to phenytoin in the control of status epilepticus and for its lower risk of drug interference with the metabolism of nimodipine. By the third day, the patient demonstrated significant neurological recovery to a GCS 13 and serial CTB showed hematoma resolution. A subsequent catheter angiogram did not reveal any intracranial vascular lesion. A valproate dose of $1200 \mathrm{mg} /$ day ( $18 \mathrm{mg} / \mathrm{kg} /$ day) was continued with satisfactory seizure control.

On day ten, the patient became progressively lethargic with a rapid decline in consciousness to a GCS of 3 over six hours. Recurrent seizures were not observed and an electroencephalogram revealed normal bilateral symmetrical $8-12 \mathrm{~Hz}$ alpha rhythms. A CTB did not reveal evidence of rebleeding or infarction. Blood tests including parenchymal liver enzymes levels (aspartate transaminase and alanine transaminase) were normal and the serum valproate level was within the therapeutic range at $423 \mu \mathrm{mol} / \mathrm{L}$ (347-693 $\mu \mathrm{mol} / \mathrm{L})$. However, the patient's serum ammonia level was markedly elevated at $378 \mu \mathrm{mol} / \mathrm{L}$ (10$47 \mu \mathrm{mol} / \mathrm{L}$ ), eight times the upper normal limit.

In view of suspected VHE, valproate was immediately discontinued. However, this led to rebound status epilepticus that required intravenous midazolam, propofol and phenytoin for control. A subsequent CTB showed diffused cerebral edema and the patient's serum ammonia level rose to $411 \mu \mathrm{mol} / \mathrm{L}$. Continuous veno-venous hemofiltration was started and the ammonia level was subsequently reduced to $84 \mu \mathrm{mol} / \mathrm{L}$. The patient failed to recover consciousness and experienced septic shock secondary to hospital acquired pneumonia. Although broad-spectrum antibiotics were administered, she developed multi-organ failure and succumbed on day thirteen of admission. A post-mortem microscopic examination of the liver failed to show features of active inflammation or drug-induced liver injury (DILI). 


\section{Discussion}

Valproate-induced hyperammonemia is a relative common phenomenon with a highly variable incidence, occurring in 16 to $100 \%$ of patients. ${ }^{2,4}$ The majority of patients with isolated elevations of ammonia are asymptomatic with normal liver function and are therefore often left unnoticed. ${ }^{4}$ In contrast, symptomatic valproate-induced hyperammonemia manifesting as encephalopathy is relatively uncommon. The symptoms of VHE are protean and include impaired consciousness, confusion, focal neurological deficit and seizures., ${ }^{2,3}$ Unfortunately, few clinicians are aware of this rare, but potentially reversible drug-related condition.

Several factors have been identified to be associated with the development of VHE. The concomitant use of sedatives and other anti-epileptic drugs has been shown to enhance valproate toxicity. ${ }^{1,2}$ In addition, patients with a documented history of hereditary or dietary carnitine deficiencies or urea cycle enzyme deficiencies are also susceptible. ${ }^{2,5}$ But apart from these factors, it is generally difficult to predict individual risk. Understanding of this complication is further limited by its idiosyncratic nature. VHE has been reported to occur in patients with a previous uneventful exposure to valproate. ${ }^{6}$ In addition, no association between the duration or dose of valproate with VHE was observed. ${ }^{4,6}$ With a maintenance dose of $18 \mathrm{mg} / \mathrm{kg} /$ day (the recommended adult daily dosages are 1000 to $2000 \mathrm{mg} ; 10$ to $30 \mathrm{mg} / \mathrm{kg}$ ) our patient did not receive a particularly high dose of valproate. Affected patients have been prescribed a wide range of valproate dosing regimens with resulting serum levels either above or within the therapeutic range as in our case. ${ }^{1,2}$

Valproate is widely known to cause DILI that is readily characterized histologically by the presence of acute hepatitis, cholestasis and steatosis. ${ }^{7}$ But evidence suggests that VHE often develops in the absence of acute liver injury, as illustrated by the normal serum liver function and post-mortem findings in our patient. ${ }^{4}$ This suggests the existence of alternate pathogenic mechanisms for hyperammonemia.

\subsection{Mechanisms for valproate-induced hyperammonemia}

Although the exact pathogenesis of VHE is not well understood, several key mechanisms involving hepatic and renal metabolic pathways have been proposed.

\subsubsection{Hepatic pathway}

Valproate is theorized to disrupt hepatic ammonia metabolism and the urea cycle through carnitine and co-enzyme A depletion. ${ }^{8}$ Carnitine, an essential co-factor in mitochondrial beta-oxidation, binds with valproate and is sequestered and then excreted. Depleted carnitine stores prevents the uptake of fatty acids into the mitochondria and limits the production of N-acetylglutamate (NAG), the required activator of the first enzyme in the urea cycle, carbamoyl-phosphate-synthetase I (CPS I). This reduction of mitochondrial beta-oxidation causes a metabolic shift to cytosolic omega-oxidation. The accumulation of omega-oxidative metabolites further reduces ammonia breakdown, notably through the inhibitory action of propionic acid on CPS I. ${ }^{9,10}$ See Fig. 1.

\subsubsection{Renal pathway}

It is hypothesized that valproate stimulates kidney tubule glutaminase that subsequently enhances glutamine uptake into renal cortical cell mitochondria. The conversion of glutamine to glutamate ultimately leads to increased ammonia production. ${ }^{10}$

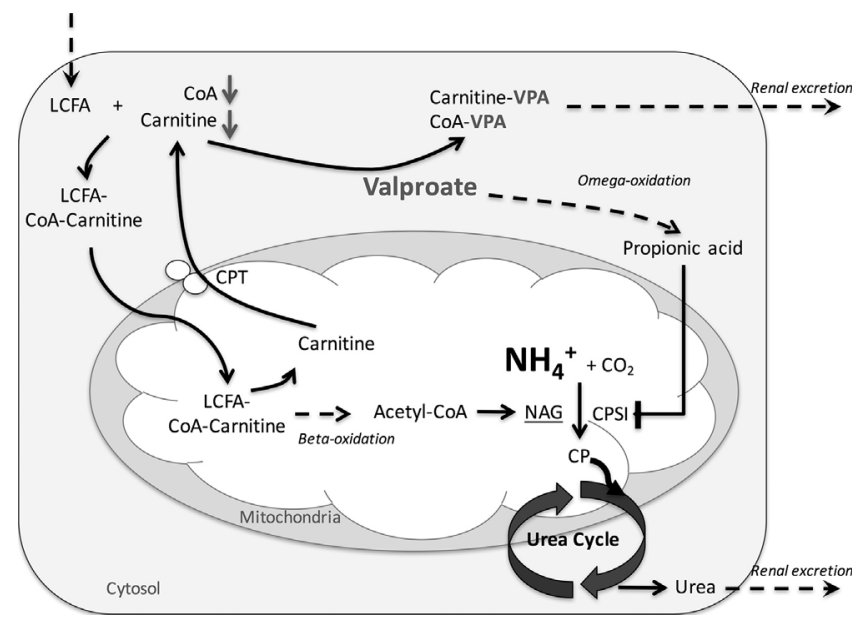

Fig. 1. Proposed mechanism for hyperammonemia due to valproic acid action in hepatocytes.

\subsection{Mechanisms for valproate-induced hyperammonemic encephalopathy}

Cytosolic ammonia, accumulated within astrocytes and neuronal cells, is first conjugated with glutatmate to form glutamine by glutamine synthetase. ${ }^{6,11}$ The derived glutamine is transported to the mitochondria where it is then reverted back to ammonia by mitochondrial glutaminase. The excess ammonia in the mitochondrial matrix leads to an increased production of reactive oxygen species, which upregulates mitochondrial permeability transition pore and cell membrane aquaporin channel expression. The oxidative stress consequently elicits to mitochondrial swelling and cytotoxic edema. ${ }^{6,11}$ The binding of ammonia with alpha-ketoglutarate can also inhibit extracellular glutamate reuptake resulting in NMDA receptor pathway mediated excitotoxicity. ${ }^{5,6,9,11}$ See Fig. 2.

\subsection{Treatment considerations}

Upon diagnosis of VHE, immediate discontinuation of valproate is mandatory and complete recovery may be possible without additional treatment. ${ }^{5}$ Founded upon the postulation that hepatic carnitine depletion contributes to valproate-induced hyperammonemia, there have been reports describing the clinical benefits of intravenous levo-carnitine supplementation in VHE patients by promoting beta-oxidation and ammonia metabolism. ${ }^{9,109,10]} \mathrm{N}$ carbamylglutamate, a synthetic analog of NAG, has also been

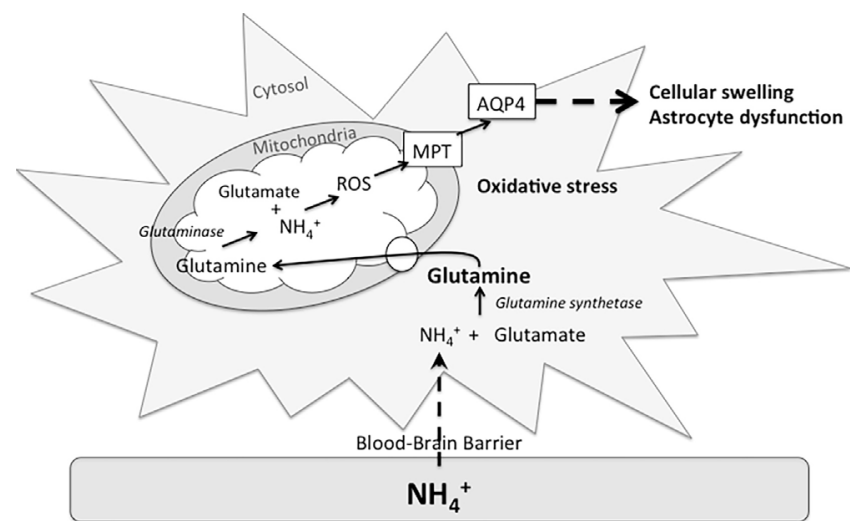

Fig. 2. Proposed mechanism of astrocyte swelling and dysfunction in hyperammonemia. 
proven to be promising by correcting downstream urea cycle NAG deficiencies. ${ }^{12}$ Others have proposed adopting comparable management strategies for hepatic encephalopathy by using lactulose, antibiotics (such as rifaximin) and dietary protein restriction to reduce intestinal flora ammonia synthesis. ${ }^{6}$ Finally, renal replacement therapy, either by hemofiltration or hemodialysis has also been shown to reduce levels of valproate and ammonia with varying degrees of success. ${ }^{6}$

\subsection{Anti-epileptic drug choices in aneurysmal subarachnoid hemorrhage}

Seizures after aneurysmal SAH occur in as many as $20 \%$ of patients and are associated with a significantly worse prognosis as they could either lead to additional neuronal injury or rebleeding of an unsecured aneurysm ${ }^{13}$. Given that our patient experienced status epilepticus, there was a clear indication to prescribe an appropriate anti-epileptic drug (AED). Valproate is routinely used in our institution for this clinical scenario since it does not induce cytochrome P450 3A4 (CYP 3A4) system enzyme activity that could alter the first pass metabolism and clearance of nimodipine. According to the American Heart Association guidelines for the management of aneurysmal $\mathrm{SAH}$, the administration of nimodipine is founded on class I, level A evidence showing a significantly reduced risk of poor neurological outcomes and delayed cerebral ischemia (DCI). ${ }^{13}$ DCI affects up to $30 \%$ of SAH patients and is the commonest determinant for long-term morbidity after ictus. The bioavailability of nimodipine can be considerably attenuated by up to 10 -fold when concomitantly dispensed with other commonly used CYP 3A4 enzyme inducing AEDs such as phenytoin. ${ }^{14}$ In addition, the American Epilepsy Society concluded, after evaluating five randomized-controlled trials, that phenytoin and valproate had similar efficacy in controlling status epilepticus, with insufficient data to support the use of levetiracetam. ${ }^{15}$ In Hong Kong, hepatitis B virus-associated liver disease is endemic with up to $10 \%$ of the population identified as chronic carriers. Given the frequency in which we encounter patients with viral chronic liver disease and SAH along with consideration of catastrophic neurological sequelae, it was believed that the benefits of prescribing valproate outweighed the risk of developing the rare complication of VHE. In retrospect, in spite of the limited evidence of its efficacy in status epilepticus, levetiracetam may have been used as an alternative.

\section{Conclusion}

Clinicians should be cognizant of the potentially fatal consequences of VHE. We recommend that any patient on valproate presenting with altered consciousness or new neurological symptoms should undergo urgent serum ammonia level testing as clinical deterioration can be rapid. VHE could develop in patients with normal liver function and therapeutic serum valproate levels. Upon establishing the diagnosis, immediate discontinuation of valproate, early administration of intravenous levo-carnitine and even renal replacement therapy in severe cases may be life-saving.

\section{Conflict of intrest}

We declare that we have no conflicting interests for the context of this case report.

\section{References}

1. Nanau RM, Neuman MG. Adverse drug reactions induced by valproic acid. Clin Biochem. 2013:46(15)1323-133810.1016/j.clinbiochem.2013.06.012 [published Online First: 20 June 2013].

2. Verrotti A, Trotta D, Morgese G, et al. Valproate-induced hyperammonemic encephalopathy. Metab Brain Dis. 2002;17(4):367-373.

3. Sousa C. Valproic acid-induced hyperammonemic encephalopathy - a potentially fatal adverse drug reaction. Springerplus. 2013;2(1)1310.1186/ 2193-1801-2-13 [published Online First: 15 January 2013].

4. Chicharro AV, de Marinis AJ, Kanner AM. The measurement of ammonia blood levels in patients taking valproic acid: looking for problems where they do not exist? Epilepsy Behav. 2007;11(3)361-36610.1016/j.yebeh.2007.06.015 [published Online First: 12 September 2007].

5. Lewis C, Deshpande A, Tesar GE, Dale R. Valproate-induced hyperammonemic encephalopathy: a brief review. Curr Med Res Opin. 2012;28(6)1039104210.1185/03007995.2012.694362 [published Online First: 6 June 2012].

6. Chopra A, Kolla BP, Mansukhani MP, Netzel P. Frye MA. Valproate-induced hyperammonemic encephalopathy: an update on risk factors, clinical correlates and management. Gen Hosp Psychiatry. 2012;34(3)290-29810.1016/ j.genhosppsych.2011.12.009 [published Online First: 2 February 2012].

7. Au J, Pockros PJ. Drug-induced liver injury from antiepileptic drugs. Clin Liver Dis. 2013;68:687-697.

8. Eubanks AL, Aguirre B, Bourgeois JA. Severe acute hyperammonemia after brief exposure to valproate. Psychosomatics. 2008;49(1):82-8310.1176/appi. psy.49.1.82.

9. Rigamonti A, Lauria G, Grimod G, Salmaggi A. Valproate induced hyperammonemic encephalopathy successfully treated with levocarnitine. $J$ Clin Neurosci. 2014;21(4)690-69110.1016/j.jocn.2013.04.033 [published Online First: 8 October 2013]

10. Mock CM, Schwetschenau KH. Levocarnitine for valproic-acid-induced hyperammonemic encephalopathy. Am J Health Syst Pharm. 2012;69(1):353910.2146/ajhp110049.

11. Bega D, Vaitkevicius H, Boland TA, Murray M, Chou SHY. Fatal hyperammonemic brain injury from valproic Acid exposure. Case Rep Neurol. 2012;4(3)224-23010.1159/000345226 [published Online First: 11 December 2012]

12. Daniotti M, la Marca G, Fiorini P, Filippi L. New developments in the treatment of hyperammonemia: emerging use of carglumic acid. Int J Gen Med. 2011;4:21-2810.2147/IJGM.S10490.

13. Connolly ES, Rabinstein AA, Carhuapoma JR, et al. Guidelines for the management of aneurysmal subarachnoid hemorrhage. Stroke. 2012;4310.1161/STR.0b013e3182587839.

14. Muck W, Ahr G, Nimodipine Kulhmann J. Potential for drug-drug interactions in the elderly. Drugs Aging. 1995;6(3):229-242.

15. Glauser T, Shinnar S, Gloss D, et al. Evidence-based guideline: treatment of convulsive status epilepticus in children and adults: report of the guideline committee of the American epilepsy society. Epilepsy Currents. 2016;16(1):48- 